\title{
Bartholin's abscess complicating food poisoning with Salmonella panama: a case report
}

\author{
A J Cummins, W A Atia
}

\begin{abstract}
A patient is presented who developed an acute Bartholin's abscess four weeks after an attack of Salmonella panama enteritis. Aspirate from the abscess also grew Salmonella panama, indistinguishable from the gut isolate in serotype and antigenic structure $(19,12: 1$, v: 1,5$)$. Some aspects of the microbiology of Bartholin's abscess and its clinical management are discussed.
\end{abstract}

(Genitourin Med 1994;70:46-48)

\section{Case report}

A 29 year old woman developed a severe enteritis with transient fevers and diarrhoea with up to six bloody stools per day during the last day of a holiday in France. She consulted her general practitioner after her return to the UK, 48 hours later, who sent a stool sample for culture and treated her with oral fluid and electrolyte replacement. He also prescribed a five day course of erythromycin $250 \mathrm{mg}$ qds to cover the possibility of infection with Campylobacter species. The stool sample, however, yielded a presumptive Salmonella species only. This isolate was sent to the Division of Enteric Pathogens, Central Public Health Laboratory, PHLS, Colindale, UK for further identification.

The patient's diarrhoeal symptoms resolved completely within seven days. Four weeks later she presented to the Genitourinary Medicine Clinic of the Royal Northern Hospital with a large $(4 \mathrm{~cm} \times 3 \mathrm{~cm}$ $\times 3 \mathrm{~cm}$ ) left-sided Bartholin's abscess.

\section{Investigations}

A standard clinical microbiological screen including microscopy and culture of specimens from the urethra and cervix for Neisseria gonorrhoeae was negative. A cervical swab for Chlamydia ELISA was reported as negative. A vaginal swab cultured only Candida sp. The contents of the Bartholin's abscess $(10 \mathrm{mls}$ of haemorrhagic pus) were aspirated under topical anaesthesia. Culture of the pus grew a Salmonella species identical in serotype, antigenic structure and antibiotic sensitivity pattern to the original stool isolate. Both isolates were identified as Salmonella panama. (1 9, 12: 1, v: 1,5). Neither gonococci nor Chlamydia trachomatis were isolated from the aspirated material.

The patient's regular partner of two years standing was invited to attend. Clinical exam- ination and investigation including a urethral swab for microscopy and Chlamydia ELISA were negative. Neisseria gonorrhoeae was not found.

Clinical course and further management

The patient was initially prescribed a one week course of oral co-trimoxazole $960 \mathrm{mg}$ bd and metronidazole $400 \mathrm{mg}$ bd, pending culture results. On follow-up five days later the affected Bartholin's gland showed some slight residual swelling; however, a second aspiration was considered unnecessary.

The Salmonella panama was reported as sensitive to co-trimoxazole; however, the patient was changed to a two week course of oral ciprofloxacin $250 \mathrm{mgs}$ tds. This agent possesses intracellular activity against Salmonella sp and was thought more likely to be effective in eradicating the organism fully from the abscess cavity, a potential reservoir for carriage.

On further follow up one week later the residual swelling had almost disappeared. Three further stool specimens were cultured at weekly intervals to exclude persistent carriage of Salmonella panama and these were reported as negative.

\section{Discussion}

We are unaware of any previous reports of a Bartholin's abscess arising as a complication of a bacterial enteritis. Also the isolation of a Salmonella sp from a Bartholin's abscess is most unusual.

The route of infection of the gland is rather conjectural. Contiguous spread by contamination of the perineal surfaces seems likely. It would be difficult to maintain adequate local hygiene in the face of repeated severe diarrhoea. However, salmonella is an invasive organism and thus haematogenous spread cannot be excluded.

A number of studies have been published on the microbiology of Bartholin's abscess. ${ }^{1-5}$ The frequency with which specific organisms infect the Bartholin's gland is variously reported. Three of these studies ${ }^{1-3}$ have given details of the bacterial species isolated from cultures of the contents of a total of 129 Bartholin's abscesses. The results are summarised in the table. The distribution of organisms suggests that the majority of the infections are caused by organisms of the perineal and vaginal flora. These may act as opportunistic pathogens.

Multiple isolates are found commonly;
Address correspondence to Dr W A Atia

Accepted for publication 13 October 1993 
Table Distribution of bacterial species recovered from pus in 129 cases of Bartholin's abscess

\begin{tabular}{|c|c|c|c|}
\hline Author & Quentin & Wren & Brook \\
\hline $\begin{array}{l}\text { No. of specimens } \\
\text { Negative samples } \\
\text { Bacteroides sp } \\
\text { Escherichia coli } \\
\text { Peptostreptococcus sp } \\
\text { Haemophilus influenzae } \\
\text { Staphylococcus aureus } \\
\text { Neisseria gonorrhoea } \\
\text { Group B streptococcus } \\
\text { Proteus sp } \\
\text { Peptococcus sp } \\
\text { Lactobacillus sp } \\
\text { Rebsiella pneumoniae } \\
\text { Streptococcus pneumoniae } \\
\text { Veillonella sp } \\
\text { Group A streptococcus } \\
\text { Citrobacter sp } \\
\text { Fusobacterium sp } \\
\text { Eubacterium sp } \\
\text { Acinetobacter sp } \\
\text { Clostridium perfringens } \\
\text { Haemophilus parainfluenzae } \\
\text { Pseudomonas aeruginosa } \\
\text { Enterobacter sp } \\
\text { Mycoplasma sp } \\
\text { Chlamydia trachomatis }\end{array}$ & $\begin{array}{r}73 \\
11 \\
13 \\
17 \\
4 \\
8 \\
6 \\
4 \\
7 \\
4\end{array}$ & $\begin{array}{r}28 \\
3 \\
15 \\
6 \\
4 \\
1\end{array}$ & $\begin{array}{r}28 \\
2 \\
23 \\
6 \\
12 \\
\\
2 \\
4 \\
3\end{array}$ \\
\hline
\end{tabular}

Brook detected $2 \cdot 8$ different species of organism per specimen in his study. Interestingly Haemophilus influenzae was isolated from eight of $73(11 \%)$ specimens in one series. ${ }^{3}$ None of these strains was capsulated, suggesting that possession of a capsule is not pre-requisite to invasion of the Bartholin's duct and gland.

The role of sexually transmitted organisms in the aetiology of Bartholin's abscess may vary according to the population studied. Rees $^{6}$ was able to recover Neisseria gonorrhoeae from the Bartholin's gland of $28 \%$ of women with gonorrhoea, most of whom were asymptomatic. However, the frequency of isolation of this organism from abscess specimens collected by percutaneous aspiration has not exceeded $15 \%$ in most recent studies.

The presence of Chlamydia trachomatis in a Bartholin's abscess was demonstrated for the first time by $\mathrm{Saul}^{7}$ in 1988: In a study by Blecker et $a l^{8}$ of 63 consecutive cases of Bartholin's abscess only one abscess was found to contain Chlamydia trachomatis. In four other cases Chlamydia trachomatis was isolated from the uterine cervix alone but not the abscess. However, the role of this organism in the pathogenesis of this condition may be indirect, for example by causing inflammation and blockage of the duct. In a selected series ${ }^{9}$ Chlamydia trachomatis has been isolated from the exudate of Bartholin's ducts from nine patients out of 30. Mycoplasma species have not been a significant finding in Bartholin's abscess in any of the studies reported.

The source of this patient's Salmonella panama was suspected by the Environmental Health Officer to have been a chicken meal consumed in a restaurant in France 24 hours before the onset of symptoms. Salmonella panama was the second most prevalent salmonella serotype in human infection in France until 1983, but in 1991 accounted for only $1.6 \%$ of human salmonella isolates sent to the national reference centre (Centre National des Salmonella et Shigella, Unité des Enterobactéries, Institut Pasteur) for identification.

We are aware of one other case report of Salmonella panama causing gynaecological pathology. The organism was cultured from pus obtained by culdocentesis from a 44 year old woman with acute salpingo-oophoritis ${ }^{10}$. Tubo-ovarian infections have also been associated with other species of salmonella, for example $S$ paratyphi $A,{ }^{11} S$ brandenburg ${ }^{12}$ and $S$ stanley. $^{13}$ Saltzman $^{14}$ reported a pelvic abscess caused by $S$ enteritidis in a patient with sickle cell trait who required extensive surgery.

There is no consensus as to the best way to manage Bartholin's abscesses. A range of methods, including aspiration, incision and drainage, marsupialisation, laser surgery and total excision have all been used. In this case the patient responded satisfactorily to a single percutaneous aspiration followed by antibiotic therapy. Aspiration may be performed using a wide bore needle (19 $\mathrm{G} \times 2$ "). Preliminary topical anaesthesia is provided by the use of lignocaine/prilocaine cream (EMLA (R)) followed by local infiltration with a minute needle.

An advantage of aspiration is that it provides an adequate specimen for microbiological investigation as well as offering immediate symptomatic relief. The culture and sensitivity results may be used to modify initial antibiotic therapy. Cheetham ${ }^{15}$ has reported a success rate of $85 \%$ in the resolution of abscesses and cysts by aspiration together with antibiotic treatment with metronidazole and penicillin (or erythromycin).

The optimal choice of antibiotic therapy for Bartholin's abscess on initial presentation will depend on clinical assessment. If there is an indication that the abscess may be gonococcal from a history of contact or findings from microscopy of genital specimens or the aspirated pus, then an appropriate antibiotic may be chosen such as amoxycillin plus probenicid or ciprofloxacin or spectinomycin. As chlamydia is frequently associated, doxycycline or erythromycin may be added.

If there is no indication as to the causative organism before culture and sensitivity results are available we suggest that a suitable broadspectrum combination might be either cotrimoxazole plus metronidazole or amoxycillin plus metronidazole.

In conclusion, thorough microbiological investigation of patients with Bartholin's abscesses makes an important contribution to the proper management of this distressing condition.

We thank Mr John Livermore, Chief MLSO, Microbiology Laboratory, Whittington Hospital for his help in identifying an unexpected organism.

1 Wren MWD. Bacteriological findings in cultures of clinical material from Bartholin's abscess. f Clin Pathol 1977;30:1025-7.

2 Brook I. Aerobic and anaerobic microbiology of Bartholin's abscess. Surg Gynecol Obst 1989;169:23-4.

3 Quentin R, Pierre F, Dubois M, Soutoul JH, Goudeau A. 
Frequent isolation of capnophilic bacteria in aspirate from Bartholin's gland abscesses and cysts. Eur $\mathcal{F}$ Clin Microbiol Infect Dis 1990;9:138-141.

4 Lee Y-H, Rankin JS, Alpert S, Daly AK, McKormack WM. Microbiological investigation of Bartholin's gland abscesses and cysts. Am $\mathcal{F}$ Obstet Gynecol 1977;129. 150-3.

5 Parker RT, Jones CP. Anaerobic pelvic infections and developments in hyperbaric oxygen therapy. $A m \mathcal{f}$ Obste Gynecol 1966;96:645-59.

6 Rees E. Gonococcal Bartholinitis. $\mathrm{Br} f$ Venereal Dis $1967 ; 43: 150-6$.

7 Saul HM, Grossman MB. The role of Chlamydia trachomatis in Bartholin's gland abscess. Am f Obst Gynecol 1988;158:576-7.

8 Blecker OP, Smalbraak DJC, Schutte MF. Bartholin's abscess: the role of Chlamydia trachomatis. Genitourin Med 1990;66:24-25.

9 Davies JA, Rees E, Hobson D, Karayannis P. Isolation of
Chlamydia trachomatis from Bartholin's ducts. $\mathrm{Br}$ Venereal Dis 1978;54:409-13.

10 Kostiala A caused by Salmonella panama and its treatment with ciprofloxacin. Case report. Br $\mathcal{F}$ Obstet Gynaecol 1989 96:120-2.

11 Michelet C, Souala F, Coulaud J P, Verliac F Paratyphoid fever A and ovarian location. Med Mal Infect 1985;15:69-72.

12 Magliulo E, Montanari M, Dietz A, Torre D. The suppurative abscess of an endometriotic ovarian cysts due to Salmonella brandenburg. Infection 1982;10:172.

13 Ghose A R, Vella E J, Begg H B. Bilateral salmonell salpingo-oophoritis. Postgrad Med $\mathcal{f}$ 1986;62:227-228.

14 Saltzman DH, Evans MI, Robichaux AG, et al. Nongonococcal pelvic abscess caused by Salmonella Nongonococcal pelvic abscess caused

15 Cheetham DR. Bartholin's cysts: Marsupialization or aspiration? Am $\mathcal{F}$ Obst Gynecol 1985;152:569-70. 